\title{
INFORMATION SECURITY AND PRIVACY
}

\author{
Tung Bui \\ Shidler College of Business \\ University of Hawaii \\ Honolulu, HI 96822 \\ tungb@hawaii.edu
}

\author{
Eric Clemons \\ The Wharton School \\ University of Pennsylvania \\ Philadelphia, PA 19104 \\ clemons@wharton.upenn.edu
}

\author{
Kevin Streff \\ The Madison Cyber Labs \\ Dakota State University \\ Madison, SD 57042 \\ kevin.streff@dsu.edu
}

The continued high profile information security breach incidents in recent years have reinforced the attention of cyber-security related issues from regulators, practitioners, and academic researchers. Despite the sustained technological progress in cyber-security, massive security breaches, unauthorized disclosure of information and the intentional misuse of private information have intensified worldwide. The purpose of this interdisciplinary minitrack is to assess the current best practices and to advance research in managing information security and privacy. This track is one of the newer mini-tracks at HICCS. In the past three years we have investigated various issues, such as the effect of policies and certification, in the management of information security and privacy in various context, such as password management and business continuity. In the future, we aim to see more research in all sectors from different perspectives, including computer security, regulations, controls, data integrity, etc. to not only provide innovative ideas in information security and privacy field but also to provide solutions to professionals.

In this third edition of this minitrack, we continue to receive a large set of quality papers leading to an acceptance rate of less than $40 \%$. As evidenced by the quality of the reviews, the selection of the papers to meet the low acceptance rate of the conference was a difficult process, with a few high quality papers not retained. We are pleased to have eight papers that cover again a wide range of topics in information security and privacy management.

Information Security Awareness: Literature Review and Integrative Framework | Lennart Jaeger

A Policy Framework for Subject-Driven Data Sharing | Mohammad Jabed Morshed Chowdhury, Alan Colman, Jun Han, Muhammad Ashad Kabir
Internalization of Information Security Policy and Information Security Practice: A Comparison with Compliance | Minjung Park, Sangmi Chai

Web Tracking - A Literature Review on the State of Research | Tatiana Ermakova, Benjamin Fabian, Benedict Bender, Kerstin Klimek

Host Inventory Controls and Systems Survey: Evaluating the CIS Critical Security Control One in Higher Education Networks | Philip Kobezak, Randy Marchany, David Raymond, Joseph Tront

Crowdsourcing Privacy Design Critique: An Empirical Evaluation of Framing Effects | Oshrat Ayalon, Eran Toch

Future Prospects of Cyber Security in Manufacturing: Findings from a Delphi Study | Katariina Kannus, Ilona Ilvonen

Should You Disclose a Data Breach via Social Media? Evidence from US Listed Companies | Pierangelo Rosati, Peter Deeney, Mark Cummins, Lisa van der Werff, Theo Lynn

We hope that the topics covered in the accepted papers for this minitrack. in spite of their inevitable and eclectic nature, and the panel discussion on future research directions that follows, provide a comprehensive view of the state-of-the-art research in information security and privacy. 